\title{
A general equation for sensory magnitude
}

\author{
WILLIAM H. ATKINSON \\ Pennsylvania State University, University Park, Pennsylvania 16802
}

\begin{abstract}
For the majority of experiments on sensory response, S. S. Stevens's power law provides a satisfactory description of the relationship between stimulus and sensory magnitudes. In its original form, it has proved to be inaccurate near the absolute threshold, when the sense organ is not in a neutral state of adaptation, when sensory noise is present, or when the sense organ is adapted to the particular stimulus under observation. This paper introduces a more general sensory law which also describes response under the conditions listed above. Stevens's power law is a special case of this more general equation. In this general formulation, sensory magnitude is considered to be the product of excitatory and inhibitory processes. Exxcitation can be described by power functions and inhibition by exponential functions. The general equation is applicable to response at both the electrophysiological and psychophysical levels.
\end{abstract}

The question of the relationship between stimulus intensity and magnitude of sensation has occupied scientists for more than a century. The first mathematical expression was introduced by Fechner (1860) when he advanced the proposition that magnitude of sensation is a linear function of the logarithm of the stimulus intensity.

Even before the turn of the century, some doubts were expressed concerning the accuracy of Fechner's law; Plateau (1872) suggested that equal stimulus ratios would generate equal sensory ratios. Nearly 50 years ago, an increased awareness began to develop that this logarithmic relationship might be inadequate; Marks (1974a) describes this development. It was during this early period that S. S. Stevens initiated a series of experiments to determine a more adequate metric for sensory magnitude; the evolution of his approach is outlined in S. S. Stevens (1975). Nearly 30 years ago, following the suggestions made by Plateau nearly a century earlier, S. S. Stevens (1953) introduced another law which stated that the logarithm of the sensation magnitude is a linear function of the logarithm of the stimulus intensity.

A host of experiments by S. S. Stevens and other researchers have demonstrated that the power law that he developed provides an accurate description of the relationship between stimulation and sensory magnitude over a wide variety of conditions; both Marks (1974a) and S. S. Stevens (1975) consider a number of these studies. Despite this agreement, however, there are many experiments in which, contrary to expectation, the power law does not hold.

Several examples of this lack of correspondence may be noted. As the threshold is approached, the

I wish to thank my wife, Ingrid, for the preparation of the figures in this paper. Requests for reprints should be sent to William Atkinson, Department of Psychology, Pennsylvania State University, University Park, Pennsylvania 16802. slope of the function relating the logarithm of the sensation magnitude to the logarithm of the stimulus intensity increases in value. Lochner and Burger (1961) and S. S. Stevens (1961a) have proposed modifications that would restore linearity. The two operations differ slightly in their effect but they are nearly equivalent. In both cases, the correction is achieved with the subtraction of a constant.

Another type of deviation is less tractable. At the higher concentrations, the magnitude of response to gustatory stimulation is less than would be expected from the power law. This departure from linearity can be observed in the paper by S. S. Stevens (1969) on scales of taste intensity; similar effects appear in research on taste by other investigators. An even more marked deviation can be observed with estimates of brightness. J. C. Stevens and S. S. Stevens (1963) have found that the power law holds when stimuli are presented for short periods of time but not when the eye is adapted to the luminance to which it is exposed. For the latter condition, the apparent brightness appears to approach an asymptote. In electrophysiological measures, the lack of correspondence with the power law is even more apparent. Atkinson $(1976,1978)$ has reported a nonmonotonic relationship between the amplitude of the click-evoked response and the intensity of the click when recordings are made from subcortical nuclei in the cat. The appearance of a nonmonotonic function is a common occurrence in auditory electrophysiology.

Moreover, even in research in which Stevens's law appears to be valid, there is a disconcerting lability in the value of the exponent. It fluctuates with the state of adaptation or in the presence of other stimulation, such as noise. If a visual stimulus is viewed in the presence of a glare source or if loudness is estimated for a tone embedded in noise, there is an apparent change in the exponent. For example, if the brightness of a light seen alone is matched to that of 
a light seen in the presence of glare, the logarithms of these two variables will appear to be linearly related, but the slope will be greater than unity; this suggests an increase of the exponent in the presence of glare. A similar effect is observed for speech floating in a background of noise. Adaptation to light or to a tactual stimulus seems to increase the value of the exponent. S. S. Stevens (1966) has designated this apparent change in the exponent as a power group transformation.

Unfortunately, the introduction of this type of transformation generates an awkward difficulty. If the exponent varies, then the ratio between sensations will also vary. For example, let us assume that two stimuli have an energy ratio of $16: 1$ and the exponent is 0.25 . Then the magnitude of one sensation will be twice that of another. Now, if we retain the same physical ratio but increase the exponent to 0.50 , then the magnitude of one sensation will be four times that of the other.

Any theory of sensory magnitude should be compatible with the principle that the balance among sensations must be maintained. Plateau (1872) established this principle in his critique of Fechner's law, and more recently Yilmaz (1967) adopted a similar position. Moreover, as S. S. Stevens (1975) has stressed, a power law is a consequence of this type of perceptual stability. Multiplicative transforms will preserve both sensory ratios and sensory distances, while linear transforms will maintain equal sense distances. However, if the exponent is permitted to vary, then both ratios and distances will be distorted by adaptation and by noise.

Since there are a number of circumstances in which the power law appears to be inadequate, it is tempting to conclude that Stevens's law is no more effective than Fechner's law in elucidating the relationship between stimulus and sensory response. However, this judgment ignores the myriad of experiments in which Stevens's law has proved to be accurate. A more fruitful approach would be to postulate that Stevens's law is a special case of a more general sensory law. To borrow an analogy from S. S. Stevens (1961b), it is not necessary to repeal his law but to amend it.

Because sensory distances and ratios are disturbed by an exponential transformation, the one critical condition for a general law is that, for a given sensory response such as loudness or brightness, the exponent will remain invariant under conditions of noise or adaptation. The first section of this paper, on excitation, will examine the evidence for the constancy of the exponent in vision and audition when the sense organ is considered to be in a neutral state of adaptation and free of the encumbrance of other stimulation. The second section, on overall state of adaptation, will show that the effect of this process can be described by a linear transform. With such an operation, magnitude differences are preserved although magnitude ratios will change in the neighborhood of the absolute threshold. The third section concentrates upon external masking. It will be demonstrated that the effect of noise is a multiplicative transform that conserves magnitude ratios. Since linear and multiplicative transforms preserve sensory distance, our perceptual world should be stable in the face of wide variations in the level of adaptation and in the presence of extraneous stimulation. The following section, on internal masking, will examine the effect of adaptation to the stimulus whose effect is being measured. It will be shown that the exponent remains invariant under this type of adaptation. In the final section, these special cases are unified and a general sensory law is constructed.

For all overall states of adaptation, excitation is a power function of the intensity of the stimulus. External masking is an exponential function of the noise-to-signal ratio. Internal masking is an exponential function of the level of the signal. If we consider excitation in the general case of any overall state of adaptation, the relationship among the variables is:

\section{Sensory magnitude}

$$
=\frac{\text { Excitation }}{\text { (External masking) (Internal masking) }}
$$

In the physical sciences, many variables are combined through the operations of multiplication and division; the relationship above is framed in a similar form. Since the time of Fechner, many researchers have sought to shape the study of sensory processes in the image of the physical sciences. The general equation for sensory magnitude that will be presented is one more step in that journey.

Most of the examples to be cited have appeared in research on audition. However, the principles to be described are common to all of the senses and their applicability to vision, touch, and taste will also be considered.

Since the purpose of this paper is the modification of the power law, let us examine it first in its pristine form. We can write:

$$
\mathrm{S}=\mathrm{rI}^{\mathrm{q}}
$$

where $S$ is the magnitude of sensory response and I is the magnitude of the physical variable. It is customary to determine the empirical constants $r$ and $q$ by plotting $\log \mathrm{S}$ as a function of $\log \mathrm{I}$; from the resultant linear equation, the values of the constants can be specified.

When stimulus values are in the vicinity of the absolute threshold, the accuracy of the power law be- 
gins to deteriorate. If the variables $\mathrm{S}$ and I are plotted on logarithmic coordinates, as the threshold is approached there is a departure from linearity with the slope of the function increasing. To describe this phenomenon, S. S. Stevens (1961a) proposed the equation:

$$
S=r\left(I-I_{0}\right)^{q}
$$

where $I_{0}$ is the absolute threshold. In the same year, Lochner and Burger (1961) suggested the equation:

$$
S=r\left(I^{q}-I_{0}^{q}\right) \text {. }
$$

Although the two equations are similar, there are important differences in their interpretation. To preserve a ratio scale on the right-hand side of Equation 2, the zero point of the physical scale has been redefined as the value of the physical variable at which sensory response disappears. Equation 3 is also consistent with the hypothesis that sensory magnitude vanishes at the absolute threshold. Marks and Stevens (1968) examine the implications of some of the techniques for correcting for the absolute threshold. When one is applying Equations 2 and 3, it is customary to estimate $I_{0}$ from the data rather than to apply an independent measure. It should, therefore, be realized that, in a mathematical sense, Equation 3 would still be valid if $S$ were scaled on an interval rather than on a ratio scale. Since, as Atkinson and Ward (1972) have demonstrated, there is some doubt that subjects always judge on the basis of sensory ratios, Equation 3 permits a greater latitude of interpretation. Throughout this paper, Equation 3 will be considered the basic equation for excitation.

\section{Exponent for Vision}

There is a generally satisfactory stability of the exponent in studies of visual response. S. S. Stevens (1970), in a review of the evidence for power functions in sensory electrophysiology, examined five studies of electrical response in Limulus. He calculated exponents that ranged from 0.21 through 0.32 . J. C. Stevens and S. S. Stevens (1963) determined the effect of a number of experimental conditions; some of this work will appear in Figure 3 when we examine the effects of adaptation. Their Table 1 lists the results of five experiments in which one of the measurements was the brightness of a pulse of light viewed monocularly with the dark-adapted eye. The mean value of the exponent was 0.29 , with a range from 0.26 through 0.32 . In a later paper, J. C. Stevens (1967) reported more precise values of 0.272 for monocular stimulation and 0.276 for binocular stimulation. Marks $(1966,1968)$ measured the size of the exponent for various locations on the retina in the dark-adapted eye and for several levels of light adaptation; his values ranged from 0.24 through 0.34 , with a mean of 0.28 . There is, therefore, a gratifying agreement with the constants calculated by Stevens (1970) for the eye of Limulus. We will encounter the value of 0.29 for the exponent for vision in Figures 3 and 7 of this paper.

\section{Exponent for Audition}

An exponent of 0.33 recurs in a number of experiments in auditory electrophysiology. Teas, Eldredge, and Davis (1962) measured the click-evoked action potential recorded from the cochlea of the guinea pig; the amplitude of the first peak is a linear function of the cube root of the sound pressure. Figure 1 depicts their results. Atkinson $(1976,1978)$ determined the peak-to-peak amplitude of the click-evoked response recorded from the superior colliculus and medial geniculate body of the cat; at low-to-moderate levels of stimulus intensity, the amplitude is again a linear function of the cube root of the sound pressure. In Figure 10, we will be discussing the results of Dallos and Wang (1974), who recorded the magnitude of the action potential in the auditory nerve of the guinea pig; for stimulus levels up to $100 \mathrm{~dB}$ SPL, the relationship between electrical response and the cube root of the stimulus pressure is linear.

The evidence for a cube root function in auditory psychophysics is somewhat controversial. It appears to be present if one calculates the cube roots of the equal loudness matches determined by Robinson and Dadson (1956). For levels below $100 \mathrm{~dB}$ SPL, the relationship between values for a particular frequency and those for the 1-kHz standard is linear. Moreover, as we will see in a later section, loudness matches made to assess the effect of a masking source also support a cube root function. On the other hand, scaling and cross-modality studies suggest an expo-

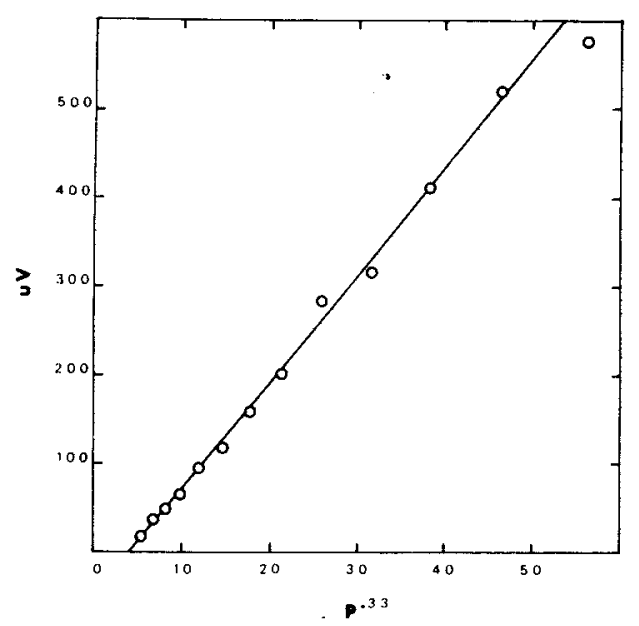

Figure 1. Amplitude of the click-evoked action potential recorded at the cochlea of the guinea pig as a function of the cube root of the sound pressure of the click. Reference level is $\mathbf{. 0 0 0 2}$ dynes $/ \mathrm{cm}^{2}$. Points were measured from Figure 11 of Teas, Eldredge, and Davis (1962). 
nent of approximately 0.60 . The exponents in scaling experiments are usually determined by plotting the logarithm of subjective loudness as a function of the logarithm of the stimulus intensity. In contrast with the narrow range found for the exponent for brightness, there is a wide variation among slopes. Marks (1974b) has catalogued the extent of this dispersion, reporting exponents that range from 0.37 to 0.85 .

The discrepancy between the exponent for the physiological and matching experiments, on the one hand, and that for the scaling studies, on the other, generates a dilemma. In vision, the exponent derived from scaling exhibits minor scatter and is in agreement with physiological studies; in audition, the exponent derived from scaling exhibits major scatter and is in disagreement with physiological studies. This suggests that it might be wise to reexamine the procedure for calculating the exponent for audition.

Since the physiological and matching experiments support an exponent of 0.33, Atkinson (1977) reanalyzed a number of these studies by plotting subjective loudness as a function of the cube root of sound pressure. In all cases, the points could be fitted with two straight-line segments with a break near $70 \mathrm{~dB}$ SPL. There is independent evidence from both physiological and psychophysical experiments that there may be a division in audition similar to the rod/ cone dichotomy in vision; they indicate that the highlevel process has a threshold near $70 \mathrm{~dB}$ SPL. The slope of the line at pressures greater than the break point is always steeper than the slope below that point, and the mean ratio of the slopes is approximately $2: 1$. However, the ratio of the slopes of the two cube-root segments is not constant. The greater the ratio, the greater would be the apparent exponent in double logarithmic coordinates. Since the scaling results can be reconciled with those from physiological and matching studies on the basis of dual processes for loudness, the exponent of 0.33 has been adopted for audition.

\section{ADAPTATION}

Although the exponent for vision seems to be stable for the dark-adapted eye and when other visual stimulation is absent, there have been reports that the exponent increases if the overall state of adaptation is altered or if visual noise is introduced. Similar effects have been reported for auditory and tactual stimulation. In this and the next section, we will review evidence suggesting that the increase in the exponent is an illusion generated by the common practice of plotting magnitude estimations on logarithmic scales.

\section{Touch}

We can begin with a report by Gescheider and Wright (1968) on the recovery of function following adaptation to a vibrotactile stimulus. Their subjects estimated the magnitude of sensation generated by particular amplitudes of vibration at intervals ranging from 5 to $360 \mathrm{sec}$ following the cessation of the adapting stimulus. When they plotted the logarithm of sensation magnitude as a function of the logarithm of the amplitude of vibration, the points for each recovery period appeared to lie on straight lines with slopes ranging from 0.64 for a 5 -sec recovery period to 0.54 for a 360 -sec recovery period.

But does the exponent really vary? The same data are plotted in Figure 2, in which subjective magnitude is expressed as a function of the square root of the vibration amplitude. The upper line is for the 360 -sec interval, and the lower is for the 5-sec interval. Both sets of points can be fit with straight lines. With reference to the form of Equation 3, adaptation produces a change in the $r$ and $I_{o}$ constants but the exponent remains invariant. The three intermediate recovery times can also be fit with straight lines. Moreover, all five recovery times conform to the simple equation:

$$
S=R\left(A^{0.50}-A_{0}^{0.50}\right),
$$

where $S$ is the magnitude of sensation, $A$ is the displacement or vibration amplitude, $A_{O}$ is the threshold displacement for a particular recovery interval, and $R$ is a constant whose value is also dependent upon the recovery interval.

The linear relationships in Figure 2 indicate that the effect of adaptation for one interval is a simple linear transform of the values for another interval. This linear transform preserves sensory distances, whereas the power group transform suggested by

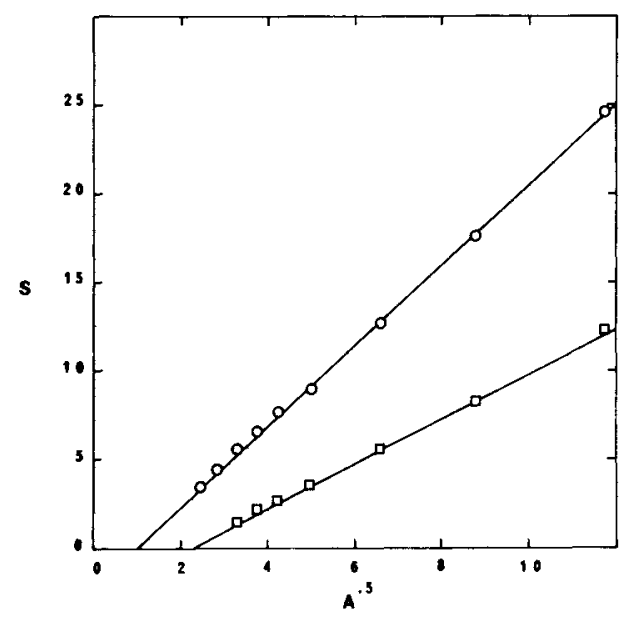

Figure 2. Magnitude of sensation as a function of the square root of the displacement of a vibrotactile stimulus. Each line is for a different period of recovery following complete adaptation. The recovery times are: squares $(5 \mathrm{sec})$ and circles $(360 \mathrm{sec})$. Original experiment is by Gescheider and Wright (1968). Polnts were mensured from Figure 5.8 of Geschelder (1976). 
Gescheider and Wright distorts both sensory ratios and sensory distances.

One other aspect of the Gescheider and Wright data is of particular interest. The five lines relating magnitude of sensation to the square root of vibration amplitude converge toward a point. In this circumstance, there will be a linear relationship between the intercept on the abscissa and the reciprocal of the slope. This means that the absolute threshold will be a function of the slope and we can write:

$$
A_{o}^{0.50}=(h / R)+j
$$

where $A_{0}$ and $R$ have the same definitions as in Equation 4 and $h$ and $j$ are empirical constants whose $a b-$ solute values are equal to the coordinates of the copunctal point.

\section{Vision}

Let us examine another study of the effects of adaptation. In a comprehensive determination of the effects of light adaptation, J. C. Stevens and S. S. Stevens (1963) compared brightness estimates made with the dark-adapted eye and with an eye adapted to $4,000 \mathrm{~mL}$. They found that the exponent appeared to increase from 0.33 to 0.47 as a consequence of adaptation to the high level of luminance. However, if the growth of subjective brightness is plotted as a function of the 0.29 power of the luminance for the two extreme conditions of adaptation, a different picture emerges. As Figure 3 shows, except for one errant point at the lowest luminance presented to the lightadapted eye, both sets of points are fit very well with straight lines. In terms of Equation 3, the effect of

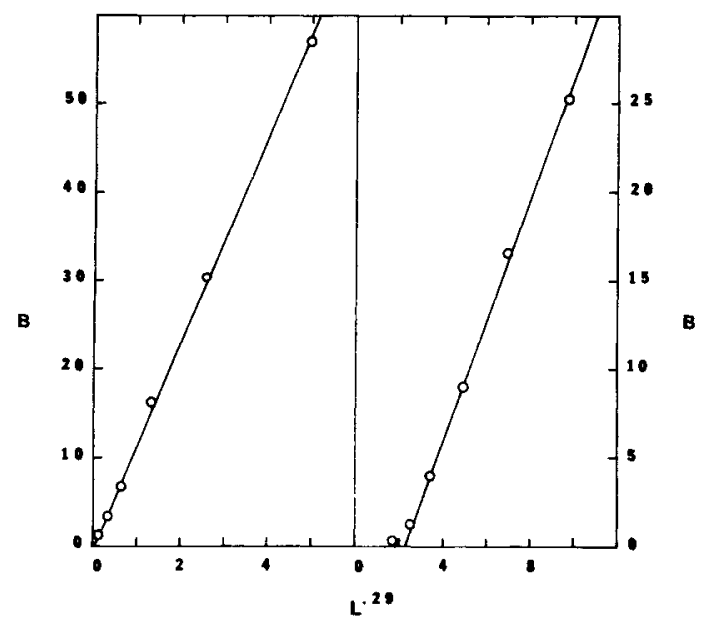

Figure 3. Brightness (B) in brils as a function of the 0.29 power of luminance ( $\mathrm{L}$ ) in millilamberts with monocular viewing using the natural pupll. Stimull were 2-sec exposures. Points in the left section were obtained with the eye adapted to complete darkness. Points in the right section were obtained with the eye adapted to $4,000 \mathrm{~mL}$. Data were measured from Figure 8 of J. C. Stevens and S. S. Stevens (1963). light adaptation is an increase in $\mathrm{I}_{\mathrm{O}}$ and a decrease in $r$; the exponent remains invariant.

Are the values of $I_{0}^{q}$ and the reciprocal of $R$ for different levels of adaptation linearly related? It is not possible to evaluate this proposition with only the two lines of Figure 3, but other data presented by Stevens and Stevens provide a test. In their Table 2, they report the results of using the method of constant stimuli to obtain interocular matches between the dark-adapted eye and an eye adapted to one of three light levels. As predicted, the 0.29 powers of the matching pairs were linearly related; the three lines also converged toward a point. Therefore, in both the visual and cutaneous senses, the effect of adaptation can be described by a simple linear transform of the equation for the unadapted sense organ; in both cases, there is a linear relationship between $\mathrm{I}_{0}^{q}$ and the reciprocal of $\mathbf{R}$.

The results reported in these two papers suggest that equal sense distances remain equal under all levels of adaptation. The special case of adaptation to the stimulus under observation requires a different type of transformation and exhibits features similar to the effects of masking. Therefore, before we consider this circumstance, let us examine a number of studies of masking.

\section{EXTERNAL MASKING}

Adaptation involves a change in the sensitivity of a sensory system. In external masking, we may consider the sensitivity to be unaffected, but the presence of one stimulus alters the response to a second stimulus. Since the interaction of two stimuli is less complex in audition than in vision, let us begin with an examination of this sense.

\section{Audition}

In the presence of a masking source, there is an elevation of the absolute threshold and a reduction in apparent loudness. As the intensity of the signal is increased, loudness grows until, at very high signal levels, the loudness of the signal under conditions of masking approaches that of the signal heard in quiet. Qualitatively similar effects are present in cases of recruitment deafness.

There have been a number of attempts to formulate a quantitative expression for the effects of a masking source. Steinberg and Gardner (1937) suggested that the effect of an external masker might be the subtraction of a constant amount of loudness irrespective of the particular level of loudness. Lochner and Burger (1961) based their formulation upon Stevens's power law.' In terms of Equation 3, they suggested that the constants $r$ and $q$ remain constant under masking but that there is a change in $\mathrm{I}_{0}$.

The proposal by S. S. Stevens (1966) is based upon Equation 2. He assumed that $r, I_{o}$, and $q$ would all 
vary with the level of the masker. Since in this formulation the exponent $q$ increases in the presence of a masking source, at high signal levels the predicted loudness of the signal under masking would exceed that of a signal heard in quiet. To circumvent this prediction, Stevens postulated a discontinuity in the function relating loudness and the intensity of the masked stimulus. Where the predicted value of the masked function equals that of the unmasked function, there is an abrupt decrease in slope.

The equation to be introduced in this section retains the basic form of Stevens's power law as expressed in Equation 3. There are, however, two important differences from Stevens's approach: the exponent remains invariant under conditions of masking, and the growth of loudness is considered to be continuous. The equation for external masking can be written:

$$
S=r\left(P_{s}^{0.33}-P_{o}^{0.33}\right) 10^{-k_{1} P_{n} / P_{s},}
$$

where $S$ is the magnitude of response, $P_{S}$ is the pressure of the signal, $P_{n}$ is the pressure of the masker, $P_{0}$ is the threshold pressure of the signal in the absence of masking, and $r$ and $k_{1}$ are empirical constants.

If $P_{n}$ is equal to zero, then the exponential term becomes unity and Equation 6 reduces to Equation 3. If $P_{n}$ is constant, then, as $P_{s}$ increases, the exponential term approaches unity and again Equation 3 will emerge; this effect occurs in cases of recruitment deafness. A third special case of particular interest is that in which the signal-to-noise ratio is maintained at a constant level; in this instance, the exponential term will be equivalent to a multiplicative constant and Equation 3 will again appear. This is similar to the effect observed with adaptation, but in the present case the threshold constant does not change.

This third condition can be tested with data from a recent paper by Hellman (1978). When the signalto-noise ratio is constant, subjective loudness should be a linear function of the cube root of sound pressure. Hellman's data, plotted in Figure 4, confirm this prediction. The striking similarity between Figure 1 and Figure 4 should be noted. The deduction tested explicitly with the data in Figure 4 can be demonstrated with the results of experiments in matching. If the loudness of a tone heard in quiet is matched to that of a tone embedded in noise set to maintain a constant signal-to-noise ratio, the cube roots of the matching pairs should be linearly related and the slope of the line should decrease as the signal-tonoise ratio becomes smaller. In a mathematical sense, this is setting equal the right-hand sides of Equations 3 and 6 . Both predictions can be confirmed with results obtained by Hellman (1978) for a $100-\mathrm{Hz}$ tone at two different signal-to-noise ratios and by Scharf (1964) for an $830-\mathrm{Hz}$ tone at four different signal-to-

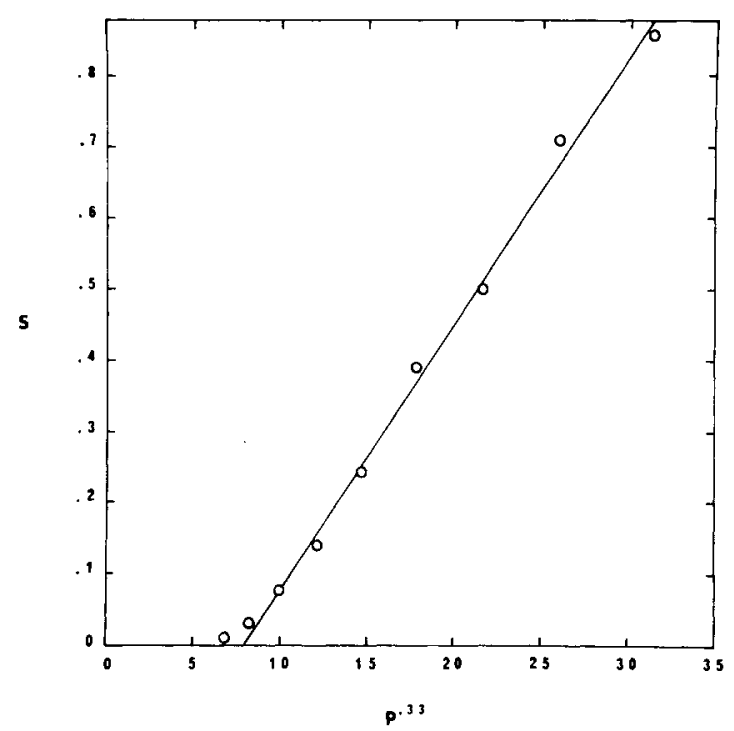

Figure 4. Relationship between the direct numerical estimation of loudness and the cube root of pressure of a $100-\mathrm{Hz}$ signal. The tone was mixed with nolse, and at each pressure a constant -10-dB S/N ratio was maintained. Data were measured from Figure 2 of Hellman (1978).

noise ratios. Hellman's data for a $250-\mathrm{Hz}$ signal with a 0-dB ratio is also linear; at $-10-\mathrm{dB}$, matches at the two highest signal levels are underestimated.

A different test of Equation 6 can be made using data reported by Pollack (1949). His listeners matched the apparent loudness of speech mixed with noise to that of speech heard under quiet conditions. For a constant signal-to-noise ratio, if the cube root of the signal pressure of the speech heard in quiet is plotted as a function of the cube root of the signal pressure of the speech heard in noise, the points lie along a straight line; it was noted earlier that the same effect is present for pure tones in the experiments by Hellman (1978) and Scharf (1964). In Pollack's data, the points for different signal-to-noise ratios form a family of linear equations that all pass through the point $(2.75$, 2.75). This estimate is only slightly larger than 1.78 , which is the cube root of Pollack's measured threshold for the detectability of speech.

The matches made by the listeners in the experiments by Hellman, Pollack, and Scharf can all be expressed by the following equation:

$$
\left(P_{m}^{0.33}-P_{o}^{0.33}\right)=\left(P_{s}^{0.33}-P_{o}^{0.33}\right) 10^{-k_{1} P_{n} / P_{s}},
$$

where $P_{m}$ is the pressure of the matching stimulus and the other symbols have the same meaning as in Equation 6. At pressures large with respect to the threshold, the logarithm of the ratio of the signal and matching pressures will be proportional to the reciprocal of the signal-to-noise ratio expressed as pressure.

In order to demonstrate that $\mathbf{k}_{\mathbf{1}}$ is a constant, we 
must take logarithms of both sides of Equation 7 . The form of equation which emerges is:

$$
\log \left(\mathrm{P}_{\mathrm{m}}^{0.33}-\mathrm{P}_{\mathrm{o}}^{0.33}\right)-\log \left(\mathrm{P}_{\mathrm{s}}^{0.33}-\mathrm{P}_{\mathrm{o}}^{0.33}\right)=-\mathrm{k}_{1} \mathrm{P}_{\mathrm{n}} / \mathrm{P}_{\mathrm{s}}
$$

This equation is plotted as the solid line in Figure 5, with $P_{0}$ equal to 2.75 and $k_{1}$ equal to 0.214 . It is based upon 87 different combinations of signal and noise, with levels of the matching stimulus ranging from 30 through $105 \mathrm{~dB}$ SPL. With only two constants in Equation 8, the standard deviation of the errors of prediction is $1.9 \mathrm{~dB}$. This is of the same order of magnitude as the error involved in measuring the illustrations in the original publication.

The study by Scharf (1964) was similar to that of Pollack, with the exception that he was investigating the loudness of pure tones rather than that of speech. As noted earlier, the cube roots of the match and masked pressures are linearly related. Since he did not investigate as many combinations of signal and noise as did Pollack, his results will not be examined to the same degree in this paper. It is worth noting that an equation similar in form to (8) is also applicable to his data with an $830-\mathrm{Hz}$ tone; the coefficient of $P_{n} / P_{s}$ is -0.076 .

A third test of Equation 7 can be made for the case in which the signal takes on a range of values but the level of the noise remains constant. In an experiment

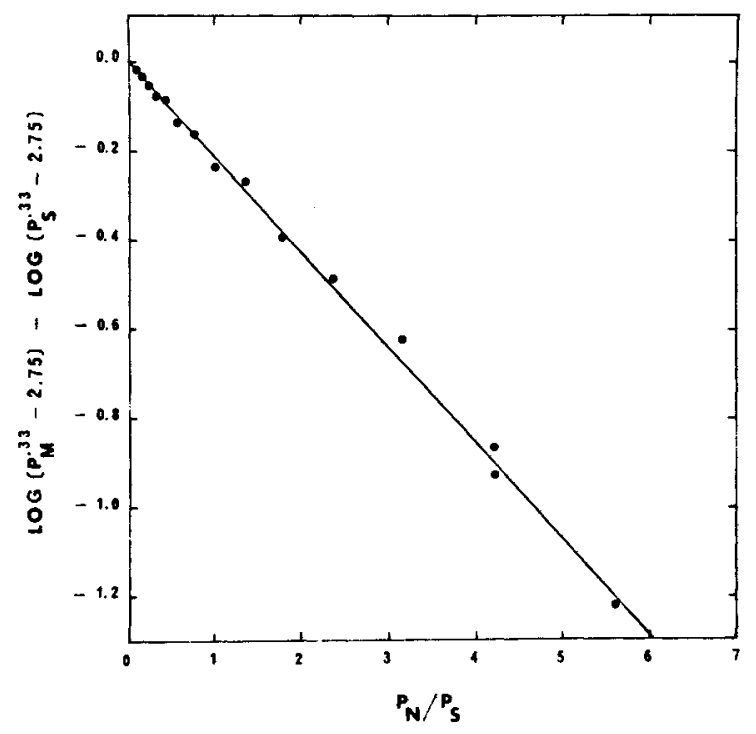

Figure 5. Units on the ordinate represent the left-hand side of Equation 8. Units on the abscissa represent the right-hand side of Equation 8. The original data were obtained by matching the loudneas of speech heard in quiet $\left(P_{m}\right)$ to the loudness of speech beard in noise $\left(P_{s}\right)$. Noise is symbolized by $\left(P_{3}\right)$, and all quantities are in terms of sound pressure. Each point is based upon four or five measurements at a particular signal-to-noise ratio with different values of signal and noise pressure. Data were measured from Figure 1 of Pollack (1949), and the values for both listeners were averaged. by Hellman and Zwislocki (1964), listeners matched the apparent loudness of a tone mixed with noise to that of a tone presented alone. In one portion of their study, $P_{n}$ was not constant for each listener but was set at a level sufficient to produce a $60-\mathrm{dB}$ threshold shift. By following a procedure similar to that employed in estimating the $\mathrm{k}_{\mathbf{1}}$ constant in the Pollack data, the value of $k_{1} P_{n}$ was estimated to be $2.66 \times 10^{3}$. Because $P_{n}$ was defined in terms of threshold shift, the value of $k_{1}$ could not be determined independently. Since their sound pressures were expressed in terms of sensation level, the $P_{0}$ constant could be set equal to 1.00. For this data, then, Equation 8 contains a single arbitrary constant. The degree to which it predicts matches is illustrated in Figure 6. The standard deviation of the errors of prediction is only $1.12 \mathrm{~dB}$. The slight reverse curvature near threshold should be noted; we will observe a similar effect in Figure 10 when voltages from an animal devoid of most of the outer hair cells in the cochlea are plotted.

This would be an appropriate point to comment upon one feature of Equation 6 which at first sight appears to be a contradiction. The term $P_{0}$ is defined as the absolute threshold in the absence of a masking source. Therefore, it seems that it would be necessary to conclude that the absolute threshold is constant even when a masker is present. However, we know from the research of Hawkins and S. S. Stevens (1950) that the threshold does increase with the level of a white noise masker. This apparent paradox can be resolved if we examine Equation 6. For a constant value of $P_{n}$, as $P_{s}$ decreases, the exponential term becomes smaller and approaches zero. We can see this process in Figure 6. When $P_{s}$ has a value of $60 \mathrm{~dB} \mathrm{SL}, P_{m}$ has a calculated value of $0.5 \mathrm{~dB} \mathrm{SL}$, which is very close to the anticipated value of $0 \mathrm{~dB} \mathrm{SL}$.

Equation 7 predicts that the value of $P_{m}$ will approach that of $P_{s}$ at very high levels of $P_{s}$; this is the familiar recruitment effect. There is, however, one experiment in which this expectation is not fulfilled. S. S. Stevens and Guirao (1967) measured the change in loudness of a $1-\mathrm{kHz}$ tone embedded in levels of white noise ranging from 50 through $100 \mathrm{~dB}$ SPL. Tone alone and tone plus noise were alternated temporally, and either $\mathbf{P}_{m}$ or $\mathbf{P}_{\mathbf{s}}$ was adjusted in separate experiments. However, at large values of $P_{3}, P_{m}$ falls consistently below $P_{s}$ for both modes of adjustment. The discrepancy becomes progressively larger at the higher levels of the noise.

The data, however, are still lawful. For each of the six conditions of noise, we can write:

$$
\begin{aligned}
\log \left(P_{m}^{0.33}-P_{o}^{0.33}\right)-\log \left(P_{s}^{0.33}-P_{o}^{0.33}\right) & \\
& =-k_{1} P_{n} / P_{s}-k_{2}
\end{aligned}
$$

This is very similar to Equation 8, with the exception of the additive constant $k_{2}$. Using a value of 1.8 for 


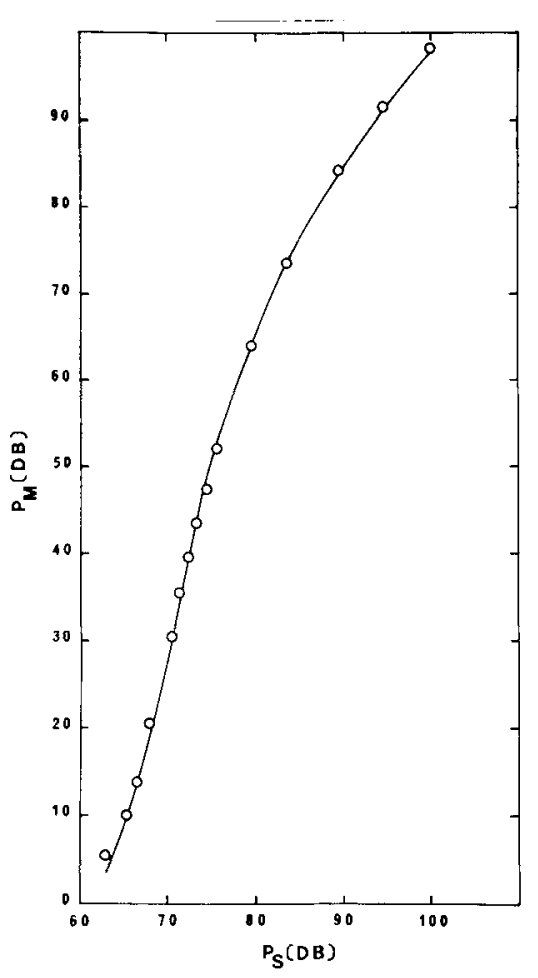

Figure 6. Equal loudness matches between a l-kHz tone $\left(P_{s}\right)$ embedded in white noise sufficient to produce a $60-\mathrm{dB}$ threshold shift and a 1-kHz tone $\left(P_{m}\right)$ heard in quiet. On both axes, $P$ is in terms of sound pressure and is specified with respect to the absolute threshold in quiet. Data were measured from the original drawing of Figure 14 in Hellman and Zwislocki (1964).

$\mathrm{P}_{\mathrm{o}}^{0.33}$, which is close to the absolute threshold for a $1-\mathrm{kHz}$ tone, Equation 9 was plotted for each of the six levels of noise. All were linear, exhibiting a degree of scatter comparable to that observed for a similar plot in Figure 5. As $P_{n}$ becomes larger, $k_{2}$ increases. A more accurate formulation of the relationship between $P_{n}$ and $k_{2}$ was not possible, since all subjects did not contribute data at all six levels.

From Equation 9, we can see that, as $P_{s}$ becomes very large, the term $k_{1} P_{n} / P_{s}$ will approach zero. However, $\mathbf{k}_{2}$ is a function of $P_{n}$ alone, so that under these conditions, $P_{m}$ will be a constant fraction of $P_{s}$. The most reasonable interpretation of $\mathbf{k}_{\mathbf{2}}$ is that it represents an adaptation to $P_{n}$. However, it must be a change that operates only during the period that $P_{n}$ is presented, since the response to $P_{m}$ is unaffected. It is possible that this represents an auditory analog to the rapid neurally mediated changes in visual sensitivity observed by Werblin (1971).

\section{Vision}

The same mechanisms that we observed in audition can also be identified in vision. Sensory magnitude is again the product of terms representing excitation, adaptation, and external masking. There are, however, two complications encountered in vision. The first is that masking is dependent, not only on the luminance of the masker, but also upon the geometrical relationships between the signal and noise sources. Horeman (1965) has demonstrated that different configurations of these sources generate marked changes in the influence exerted by the noise upon the signal. The second is that the noise source not only inhibits the signal source but also contributes to the excitatory process. Excitation is usually engulfed by its inhibitory twin, but when the source luminance is low, the noise will produce an enhancement in the apparent brightness of the source. This elevation can be discerned in the results obtained by Heinemann (1955) and in the replication of his research by Horeman. This concept of concurrent excitatory and inhibitory processes was proposed by Rodieck and Stone (1965) in their analysis of receptive fields in retinal ganglion cells of the cat. And later, when we examine the processes of internal masking, this concept will reappear.

In vision, then, sensory magnitude is the sum of two processes. It will simplify the discussion if we consider each separately before evaluating the effect of their sum. The first process can be written:

$$
\mathrm{S}_{1}=\mathrm{r}\left(\mathrm{L}_{\mathrm{s}}^{0.29}-\mathrm{L}_{\mathrm{o}}^{0.29}\right) 10^{-\mathrm{K}_{1} \sqrt{\mathrm{L}_{\mathrm{n}} / \mathrm{L}_{\mathrm{s}}},}
$$

where $S$ is the magnitude of sensory response, $L_{s}$ is the luminance of the source, $L_{n}$ is the luminance of the noise, $L_{o}$ is the absolute threshold in the absence of noise, and $r$ and $K_{1}$ are empirical constants. $K_{1}$ varies with the geometrical relationships between the signal and noise sources.

There are strong similarities between Equation 10 for vision and Equation 6 for audition. $\mathrm{L}_{\mathrm{s}}^{0.29}-\mathrm{L}_{\mathrm{o}}^{0.29}$ is the analog of $\mathbf{P}_{\mathbf{s}}^{0.33}-\mathbf{P}_{0}^{0.33}$. In the exponential, the term $\sqrt{L_{n} / L_{s}}$ corresponds to $P_{n} / P_{s}$. However, in vision, the exponent is the square root of the ratio of the two luminances rather than the simple ratio we found in audition. The stimulus in vision is usually expressed in terms of energy, which is the square of pressure. Since $L_{n}$ and $L_{s}$ in Equation 10 are in terms of energy, the radical sign means that masking in vision is also an exponential function of the ratio of the noise and signal pressures.

For the second process we can write:

$$
S_{2}=r\left(K_{3} L_{n}^{0.29} \sqrt{L_{n} / L_{s}}\right) 10^{-K_{1} \sqrt{L_{n} / L_{s}},}
$$

where the terms have the same definition as for Equation 10 and $\mathrm{K}_{3}$ is an empirical constant. As in Equation 10, we can express sensory magnitude as the product of excitatory and inhibitory processes. However, with the second component, the excitatory term is a fraction of the luminance of the noise source. If this fraction were constant, the effect might be attributed to the presence of scattered light. However, the fraction is also a function of the noise-to- 
signal ratio. As the source luminance becomes large, the fraction diminishes to zero. This accords with the observation that the enhancement effect disappears at the higher source luminances.

The equation for visual brightness in the presence of a masking source can therefore be written as:

$\mathrm{S}=\mathrm{r}\left(\mathrm{L}_{\mathrm{s}}^{0.29}-\mathrm{L}_{\mathrm{o}}^{0.29}+\mathrm{K}_{3} \mathrm{~L}_{\mathrm{n}}^{0.29} \sqrt{\mathrm{L}_{\mathrm{n}} / \mathrm{L}_{\mathrm{s}}}\right) 10^{-\mathrm{K}_{1} \sqrt{\mathrm{L}_{\mathrm{n}} / \mathrm{L}_{\mathrm{s}}} .}$

The similarities between Equation 6 for audition and Equation 12 for vision should be noted. In both cases, sensory magnitude is proportional to the product of excitatory and inhibitory processes. The major difference is that, in vision, the excitatory process also contains a component contributed by the noise source.

In the four experiments on vision that we will examine, $L_{s}$ and $L_{n}$ were presented to one eye and a matching luminance $\mathrm{L}_{\mathrm{m}}$ was presented to the other eye. An equation for vision similar to Equation 7 for audition can be written:

$$
\begin{aligned}
& \left(L_{m}^{0.29}-L_{o}^{0.29}\right) \\
& \quad=\left(L_{s}^{0.29}-L_{o}^{0.29}+K_{3} L_{n}^{0.29} \sqrt{L_{n} / L_{s}}\right) 10^{-K_{1} \sqrt{L_{n} / L_{s}}} .
\end{aligned}
$$

For a constant value of $L_{n}$, as $L_{s}$ becomes larger, the ratio $L_{n} / L_{s}$ approaches zero. Under this condition, not only does the excitation contributed by $L_{n}$ diminish toward zero, but the value of the exponential term approaches unity. There is, therefore, an effect in vision similar to recruitment in audition. This process can be observed in the papers by Diamond (1953) and by Heinemann (1955), in which the masking effect becomes progressively weaker at the higher source luminances.

There is no simple procedure for the evaluation of the constants $K_{1}$ and $K_{3}$. The approach adopted here is first to estimate $L_{0}$, which can vary only within narrow limits imposed by the data. Then trial values of $K_{3}$ are inserted in Equation 13 and logarithms are taken of both sides of the equation to determine $K_{1}$.

At first sight, Equation 13 appears to be complex, but under certain conditions it reduces to a simple form. If $\mathrm{L}_{\mathrm{n}} / \mathrm{L}_{\mathrm{s}}$ is a constant, then $\mathrm{L}_{\mathrm{m}}^{0.29}$ will be a linear function of $\mathrm{L}_{\mathrm{s}}^{0.29}$. Therefore, the subjective brightnesses for the matching and masked sources will be linearly related. In the introduction, it was proposed that the touchstone for any equation of sensory magnitude should be the preservation of equal sense distances or equal sense ratios under conditions of adaptation or masking. Equation 13 fulfills this requirement. The effect of constant noise-to-signal ratios in vision was determined by Diamond (1953); his results exhibit the predicted linearity.

We will return to Diamond's (1953) paper shortly, but let us first examine a study by S. S. Stevens and
Diamond (1965), since we must build upon the relationships present in this body of data. The signal and noise sources were very small squares, $6.5 \mathrm{~min}$ on a side. There were 14 angles of separation coupled with 9 levels of source luminance for a total of 100 conditions. The noise luminance was constant at $4.8 \log \mathrm{mL}$.

Since their noise source was at an extremely high level of luminance, some adaptation was present. Earlier, we noted the same type of effect in audition in the results of S. S. Stevens and Guirao (1967). Therefore, we must add one more constant to Equation 13 and write:

$$
\begin{aligned}
& \left(\mathrm{L}_{\mathrm{m}}^{0.29}-\mathrm{L}_{\mathrm{o}}^{0.29}\right) \\
& \quad=\left(\mathrm{L}_{\mathrm{s}}^{0.29}-\mathrm{L}_{\mathrm{o}}^{0.29}+\mathrm{K}_{3} \mathrm{~L}_{\mathrm{n}}^{0.29} \sqrt{\mathrm{L}_{\mathrm{n}} / \mathrm{L}_{\mathrm{s}}}\right) 10^{-\mathrm{K}_{\mathrm{t}} \sqrt{\mathrm{L}_{\mathrm{n}} / \mathrm{L}_{s}}}-\mathrm{K}_{2} .
\end{aligned}
$$

$\mathrm{L}_{0}^{0.29}$ has a value of 0.32 ; the measured thresholds were 0.29 with the glare source absent and 0.34 with the glare at its greatest angle of separation. The $\mathrm{K}$ constants are all proportional to the reciprocal of the tangent of the angle separating the two sources. The three equations can be written:

$$
\mathbf{K}_{1}=\frac{\mathbf{k}_{1}}{\tan \theta}, \mathbf{K}_{2}=\frac{\mathbf{k}_{2}}{\tan \theta} \text {, and } K_{3}=\frac{\mathbf{k}_{3}}{\tan \theta} .
$$

Let us first examine the adaptation term that is a function of $\mathrm{K}_{2} ; \mathbf{k}_{2}$ is equal to $1.50 \times 10^{-3}$. It would be anticipated that $k_{2}$ is an increasing function of $L_{n}$ and probably also of the size of the source. There are no visual data to evaluate these possibilities, but in the experiment by S. S. Stevens and Guirao (1967), $k_{2}$ is an increasing function of $P_{n}$ in audition. As the angle of separation increases, $\tan \theta$ becomes larger and $K_{2}$ decreases. As $\theta$ approaches $90 \mathrm{deg}, K_{2}$ approaches zero and the exponential approaches unity. In other words, the adaptation effect decreases as the angle between the $\mathrm{L}_{s}$ and $\mathrm{L}_{n}$ sources becomes larger.

A similar effect is present with the $\mathbf{K}_{1}$ constant; $\mathrm{k}_{1}$ is equal to $5.98 \times 10^{-5}$. A study by Diamond (1955) suggests that $K_{1}$ increases with the area of the noise source, although the report also reveals that large individual differences are present. The only experiment to consider the effect of the area of the signal source is by Diamond (1962), and it indicates that this variable is of negligible influence. As the angle of separation increases, we have the same type of change observable with adaptation. When $\theta$ is equal to $90 \mathrm{deg}$, the exponential has a value of unity.

As $\tan \theta$ becomes very large, $K_{3}$ also approaches zero, and, as a consequence, the excitatory component from $L_{n}$ also approaches zero. The constant $k_{3}$ 
has a value of $4.04 \times 10^{-6}$. Therefore, at very large angles, Equation 14 will be a function of excitation alone.

How well are the results of S. S. Stevens and Diamond predicted by Equation 14 ? With only the three $k$ constants free to vary, the standard deviation of the errors of prediction of the 100 matches is only $1.04 \mathrm{~dB}$; this is comparable to the accuracy in audition attained with the results by Pollack (1949) and by Hellman and Zwislocki (1964). If the five cases at $.06 \mathrm{~mL}$, the lowest signal luminance, are omitted from the calculations, the error of prediction drops to $.86 \mathrm{~dB}$.

In their experiment, the independent variables were the signal luminance and the angle of separation between the signal and the noise. Since the noise luminance was constant, we have still not demonstrated that the terms containing $L_{n}$ in Equations 12, 13, and 14 are correct. What we now need is a set of data in which the angle between the sources is constant and $\mathrm{L}_{\mathrm{n}}$ is varied. Diamond (1953) used 33-min square sources placed next to each other and varied both $\mathrm{L}_{s}$ and $L_{n}$. There were seven levels of $L_{s}$ ranging from -.39 to $2.71 \log \mathrm{mL}$, and the values of $\mathrm{L}_{\mathrm{n}}$ were selected to form a limited set of $L_{n} / L_{s}$ ratios. It will be recalled that according to Equation 13, if this ratio is constant, then $\mathrm{L}_{\mathrm{m}}^{0.29}$ will be a linear function of $\mathrm{L}_{\mathrm{s}}^{0.29}$; Diamond's data confirm this expectation.

In the S. S. Stevens and Diamond experiment, the signal and noise sources were viewed with the left eye and the match source by the right eye. In the absence of noise, $\mathrm{L}_{\mathrm{m}}$ was consistently set to be slightly less than $L_{s}$, so the tabulated values of $L_{m}$ were adjusted to correct for this imbalance. In Diamond's experiment, $L_{m}$ was on the left and was adjusted by the subject to be slightly more than $\mathrm{L}_{\mathrm{s}}$. Both experiments suggest that, for equal source luminances, the one seen by the left eye will appear slightly less bright than the one seen by the right.

To analyze Diamond's data, it was therefore necessary to multiply $\mathrm{L}_{\mathrm{m}}^{0.29}$ by the constant 0.952 to align the brightnesses seen by the two eyes. When this is done, Equation 13 provides a satisfactory fit, with $L_{0}^{0.29}$ equal to $0.20, k_{1}$ equal to $7.8 \times 10^{-4}$, and $k_{3}$ equal to $4.8 \times 10^{-4}$. Both of these constants are larger than their counterparts in the S. S. Stevens and Diamond experiment. Since the area of the sources in the Diamond study was approximately 26 times that of those in the S. S. Stevens and Diamond work and since the Diamond (1955) study showed that the inhibitory effect increases with the area of the $L_{n}$ source, it seems reasonable to conclude that the contribution of $L_{n}$ to excitation is also a function of the area of the noise source. For the present study by Diamond, the standard deviation of the errors of prediction is $1.25 \mathrm{~dB}$ for all 60 measurements and $.99 \mathrm{~dB}$ if the data for the $-.39 \log \mathrm{mL}$ condition is omitted. The error is slightly larger than that for Stevens and Diamond, but it should be noted that data for a single subject were used in this study.

In the preceding pair of experiments, $\mathrm{L}_{\mathrm{s}}$ was one of the independent variables and $\mathrm{L}_{m}$ was the dependent variable. In the next pair by Fry and Alpern (1953) and Leibowitz, Mote, and Thurlow (1953), the independent variables are $L_{n}$ and the angle of separation between signal and noise sources. However, $\mathrm{L}_{m}$ is fixed at a single value in each experiment and $L_{s}$ is the dependent variable.

The experiment by Leibowitz, Mote, and Thurlow used apparatus similar to that employed by Diamond. $\mathrm{L}_{\mathrm{m}}$ was maintained constant at $1 \mathrm{~mL}$, and the subject adjusted $L_{s}$. Stimuli were squares $30 \mathrm{~min}$ on a side, with six center-to-center separations ranging from 30 through $570 \mathrm{~min}$. There were 10 levels of $L_{n}$, ranging from 0 through $400 \mathrm{~mL}$.

The area of the sources was 21.3 times larger than those in the Stevens and Diamond study. Despite this difference, in agreement with what Stevens and Diamond had observed, the inhibitory effect wanes for 30 through 40,60 , and $90 \mathrm{~min}$ of separation. However, when the distance changes from 90 to $210 \mathrm{~min}, \mathrm{~L}_{\mathrm{s}}$ increases for all values of $L_{n}$, and at $570 \mathrm{~min}$ it is still higher than for $90 \mathrm{~min}$ at the larger values of $L_{n}$. This inversion does not appear in the data of Stevens and Diamond.

Equation 13 is valid for all six separations. Moreover, for the first four, the $L_{o}$ and $k$ constants are the same and are very similar to those obtained by Diamond (1953). $\mathrm{L}_{\mathrm{o}}^{0.29}$ is again equal to $0.20, \mathrm{k}_{1}$ is $7.2 \times 10^{-4}$, and $\mathrm{k}_{3}$ is equal to $4.4 \times 10^{-4}$.

If we exclude the two largest separations from the analysis, we can calculate the accuracy of fit. Since $L_{s}$, the independent variable, is an implicit function of $L_{n}$ and $\theta$, we cannot easily calculate the accuracy of prediction of $L_{s}$. However, if we substitute the constants in Equation 13, the right-hand side should be equal to 0.800 . The mean value of the constant is 0.792 with a standard deviation of 0.066 .

In the experiment by Fry and Alpern (1953), the sources were vertical rectangles 106.5 times greater than those used by Stevens and Diamond. There were two noise sources flanking the signal. They present data for only two subjects, who could not be combined since different values of $L_{n}$ were used for each. A preliminary analysis suggests that Equation 13 would also be valid for their results with constants comparable to those in the Diamond and in the Leibowitz, Mote, and Thurlow studies. However, the variability of the subjects is too great to permit an accurate estimate.

The $L_{s}$ and $L_{n}$ sources are usually white light. This raises the question of whether the $S_{2}$ component would be present if $L_{s}$ and $L_{n}$ were complementary hues. In a similar vein, one can conjecture that the chromatic cancellation that occurs when complements are mixed may be another example of visual masking. 


\section{Taste}

Masking is also a multiplicative function in taste. Beebe-Center, Rogers, Atkinson, and O'Connell (1959) reported data on masking in solutions of sucrose and sodium chloride. For a given concentration of sodium chloride, the matching concentration of sucrose is always a constant percentage of the sucrose concentration in the mixture. A similar effect is present for a given concentration of sucrose as masker. This type of relationship is similar to that noted earlier for high levels of speech with a constant noise-tosignal ratio. However, it should be noted that, in taste, masking is a function of the concentration of the noise component alone rather than of the ratio of noise-to-signal concentrations.

Similar conclusions can be drawn from a more recent study by Moskowitz (1971). His subjects performed magnitude estimations for the four basic tastes: sweet, sour, salty, and bitter. The judgments were made for the basic tastes in isolation and in pairs. His results suggest that a particular masking concentration reduces the magnitude of taste by the same percentage at all concentrations of the signal. One inference that can be drawn from both studies is that an effect similar to recruitment does not occur in taste. However, Beebe-Center, Rogers, Atkinson, and $O^{\prime}$ Connell suggest that masking in taste may also be an exponential function; this possibility could not be evaluated with the data of Moskowitz.

\section{INTERNAL MASKING}

There are similarities between internal masking, on the one hand, and both adaptation and external masking, on the other. In common with adaptation, internal masking involves a reduction in sensitivity. However, internal masking is adaptation to the particular stimulus being observed. In common with external masking, internal masking is an exponential function of the masker level.

In the experiment illustrated in Figure 3, subjects were adapted either to darkness or to $4,000 \mathrm{~mL}$. The stimuli to be judged were brief flashes of light which did not alter the state of adaptation. However, when subjects are adapted to the stimuli to be evaluated, the linearity evident in Figure 3 does not emerge.

J. C. Stevens and S. S. Stevens also measured apparent brightness when the subject was adapted to the stimulus; their results are depicted in the righthand section of Figure 7. Since they employed a natural pupil, their luminance values were converted to trolands using the pupil areas measured by Reeves (1920). The solid line is an expression of the equation:

$$
\mathrm{B}=\mathrm{rL}^{0.29} 10^{-\mathrm{c} \sqrt{\mathrm{L}}} \text {, }
$$

where $B$ is the subjective brightness in brils, $L$ is the retinal illuminance in trolands, $\mathrm{c}$ is an empirical con-

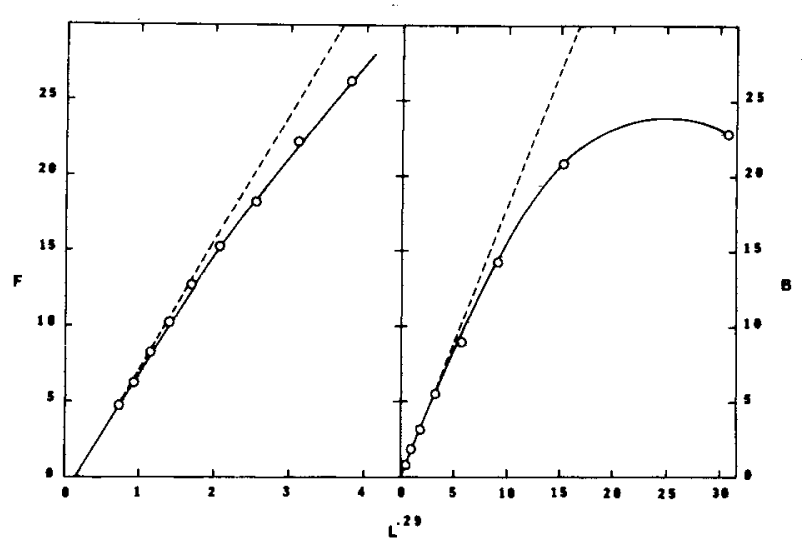

Figure 7. Left-hand section depicts the impulse frequency in a single fiber of Limulus as a function of the 0.29 power of the relative luminance. Counts were made between 15 and $20 \mathrm{sec}$ after the onset of light stimulation. Measurements were made from Figure 6 of Fuortes (1958) using the unit denoted by solid lines in that illustration. The solid line in the present illustration is a theoretical function described by Equation 16. The right-hand section portrays the brightness in brils of a source to which the human eye was adapted during the estimation. Since the original measurements had been made with a natural pupil, the light levels presented to the eye have been converted into trolands. The units on the abscissa are trolands to the $\mathbf{0 . 2 9}$ power. The data points were measured from Figure 7 of J. C. Stevens and S. S. Stevens (1963). The solid line is a theoretical function described by Equation 15.

stant, and $r$ is a constant to match the units of $B$. It should be noted that the same exponent value, 0.29 , is used in Figures 3 and 7.

The left-hand section of Figure 7 depicts the response of Limulus under steady state conditions. Spike frequency is expressed as a function of luminance to the 0.29 power. These measurements were made by Fuortes (1958) over a period of 15 to $20 \mathrm{sec}$ after the onset of the stimulus. The response is linear for the lower luminances, but it departs from linearity at the higher levels. The solid line is an equation which exhibits the form:

$$
\mathrm{F}=\mathrm{r}\left(\mathrm{L}^{0.29}-\mathrm{L}_{\mathrm{o}}^{0.29}\right) 10^{-\mathrm{c} \sqrt{\mathrm{L}}},
$$

where $F$ is the frequency of discharge, $L$ is the luminance of the stimulus, $L_{0}$ is the threshold luminance of the stimulus, $\mathrm{c}$ is an empirical constant, and $r$ is a constant used to match the units of $F$. Equation 16 has exactly the same form as Equation 15, except for the threshold constant which could not be estimated for the J. C. Stevens and S. S. Stevens data.

It is interesting to note that the exponent of 0.29 holds for both Limulus and for humans. As in Equations 10-14, the exponential is a function of the square root of luminance, since the units are expressed in terms of energy. Equations 15 and 16 are similar in a mathematical sense to Equation 11. If $L_{n}$ in Equation 11 is held constant, then there will be a value of $\mathrm{L}_{\mathrm{n}} / \mathrm{L}_{\mathrm{s}}$ where $\mathrm{S}$ is a maximum. 


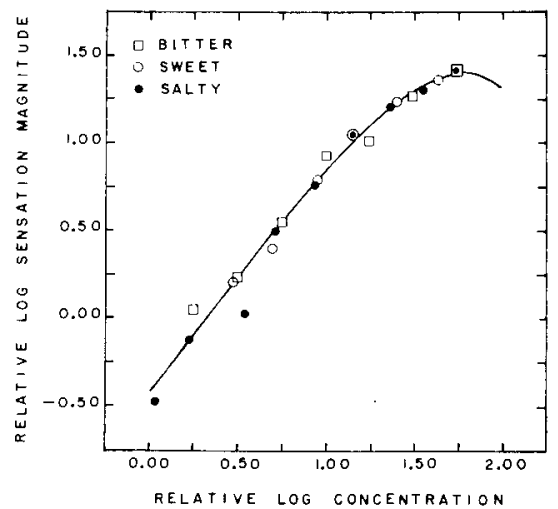

Figure 8. Relative $\log$ sensation magnitude as a function of relative log concentration. The values for the three basic tastes have been converted to a common scale by adding constants to the log sensation magnitudes and to the log concentrations for sodium chloride and for quinine sulfate. The solid line is a theoretical function which appears as Equation 17 in the text. Data points were measured from Figures 3, 8, and 10 of S. S. Stevens (1969).

\section{Taste}

In many studies of taste, when the logarithm of magnitude of response is plotted as a function of the logarithm of concentration, the upper portion of the curve will appear to be negatively accelerated rather than linear. This decrement can be observed in the reports by Bartoshuk and Cleveland (1977), Borg, Diament, Strom, and Zotterman (1967), Moskowitz (1970), Pfaffman (1955), and S. S. Stevens (1969). Attenuated response is observed at high concentrations for both judgments of sensory magnitude and for the electrical response of the gustatory nervous system in rats and humans. Although it is possible that this waning could be a consequence of physical factors with sucrose such as viscosity, it also appears when sodium chloride is the stimulus. Figure 8 depicts some of the results obtained by S. S. Stevens (1969) for three basic tastes. The solid line has the mathematical form:

$$
\mathrm{S}=\mathrm{rW}^{\mathrm{i.33}} 10^{-\mathrm{cW}}
$$

where $S$ is the relative subjective magnitude of taste and $W$ is the relative concentration in grams per $100 \mathrm{cc}$ of water. The constant $r$ matches the units of $\mathrm{S}$, and $\mathrm{c}$ is an empirical constant.

\section{Audition}

To understand Equations 15, 16, and 17, it is necessary to return to the section on external masking and to analyze Equation 6 . This equation can be considered to be the product of two processes. One, which is excitatory, is a linear function of the cube root of the pressure of the stimulus; Equation 3 expresses the pure excitatory process. The other, which is inhibitory, is an exponential function of the ratio of the noise-to-signal pressure. In the preceding section, it was demonstrated that visual and auditory noise would attenuate the response to a stimulus. Since adaptation to the stimulus has a similar effect, it is reasonable to suspect that a similar mechanism may be involved.

Figure 9 illustrates the processes involved in responding to an auditory stimulus to which the organism is adapted. The straight line in the upper lefthand corner portrays an excitatory process which is a linear function of the cube root of the sound pressure; this is Equation 3. The curved line in the lower left-hand corner illustrates an inhibitory process which is an exponential function of the sound pressure. The product of these two processes produces the nonmonotonic function on the right. This form of curve is quite common in auditory electrophysiology, and Atkinson $(1976,1978)$ has demonstrated its applicability to subcortical structures in the cat. The equation introduced in these papers is:

$$
\mathrm{V}=\mathrm{r}\left(\mathrm{P}^{0.33}-\mathrm{P}_{\mathrm{o}}^{0.33}\right) 10^{-\mathrm{cP}},
$$

where $\mathrm{V}$ is the peak-to-peak voltage of the clickevoked response and $P$ is the pressure of the stimulus. The constants $r$ and $c$ were defined in Equation 15.

Equation 18 is not limited to click stimulation or to the higher levels of the auditory nervous system in the cat. Figure 10 depicts the magnitude of the action potential in the auditory nerve of the guinea pig recorded by Dallos and Wang (1974) using an 8-kHz tone burst. For the moment, we can confine our attention to the data represented by circles, which were recorded from an animal with a normal cochlea. The

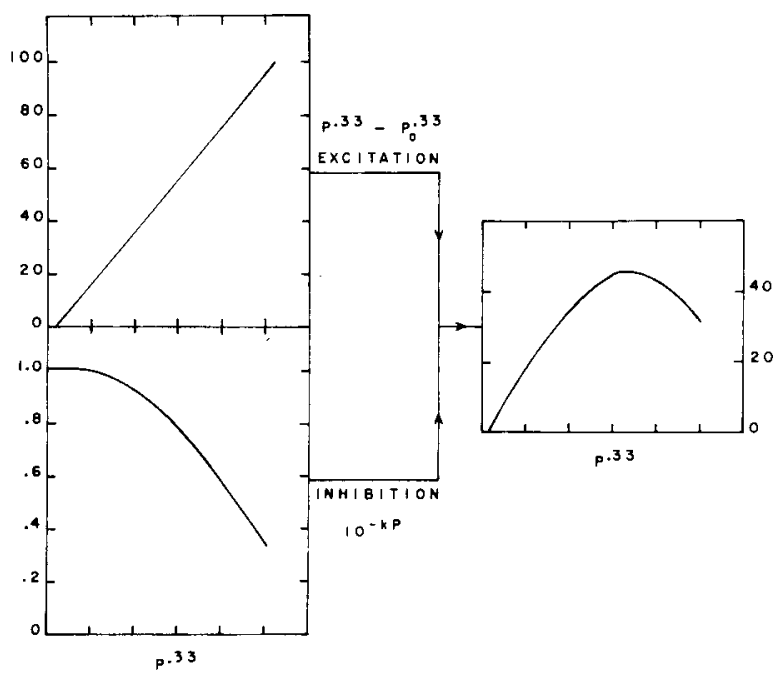

Figure 9. Theoretical components of response to stimulation to which the organism is adapted. The product of the excitatory and inhibitory processes on the left generates the nonmonotonic curve on the right. This is the type of function described in the text by Equations 15, 16, 17, 18, and 20. 


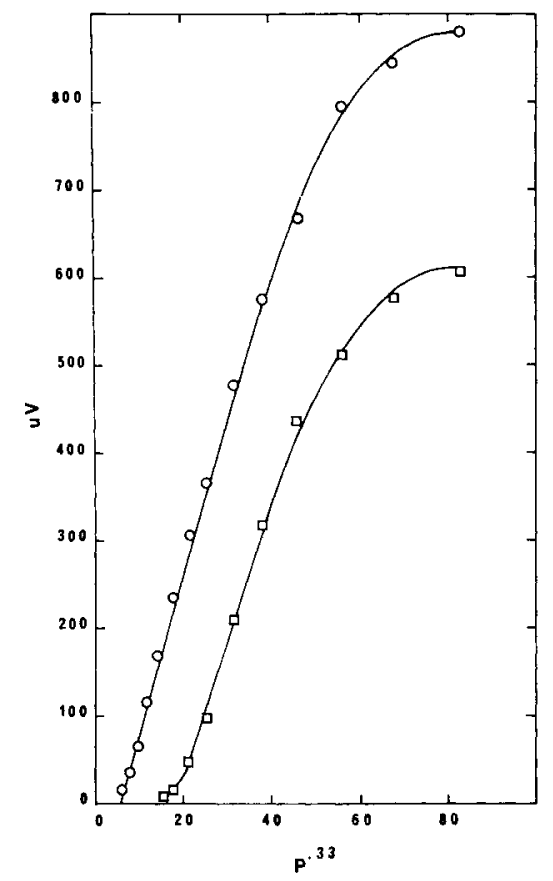

Figure 10. Action potential of the auditory nerve recorded from the cochlea of the guinea pig. The stimulus was an $8-\mathrm{kHz}$ tone burst. The circles represent the voltages from an animal with a normal cochlea, and the squares denote the voltages from an animal in which the majority of the outer hair cells in the basal turn had been destroyed. Units on the abscissa are the cube roots of the sound pressure of the bursts. The solid lines are theoretical functions described by Equations 18 and 19 . Data were measured from Figure 5 in Dallos and Wang (1974).

solid line through these points is Equation 18, with $\mathrm{r}$ equal to $18, \mathrm{P}_{0}^{0.33}$ equal to 6 , and $\mathrm{c}$ equal to $0.36 \times 10^{-6}$. Except for the four highest pressures, which ranged from 100 through $115 \mathrm{~dB}$ SPL, the relationship between electrical response and the cube root of pressure is linear; as noted earlier, in Figure 1, the same function appears for the same species in the data of Teas, Eldredge, and Davis (1962).

Equations 6 and 18 differ only in the exponential term. In the auditory nervous system of the cat and the guinea pig, the signal masks itself. In human psychophysical judgments in vision and audition, the masker is the ratio of the noise and signal pressures. The formal similarity between Equations 6 and 18 reinforces the hypothesis that internal and external masking may be a function of similar mechanisms.

The lower curve in Figure 10 represented by squares provides a clue for the mechanism of auditory masking. The points were derived from an animal in which most of the outer hair cells of the cochlea had been destroyed by the drug Kanamycin; the inner hair cells remained intact. If we compare voltages at the same sound levels for normal and Kanamycin-treated animals, we can write an equation of the form:

$$
\log V_{t}-\log V_{u}=-k_{1} / P_{s}-k_{2} \text {, }
$$

where $V_{t}$ and $V_{u}$ are tone-evoked voltages from the Kanamycin treated and untreated animals, respectively, $P_{s}$ is the pressure of the stimulus, and $k_{1}$ and $k_{2}$ are empirical constants. In a formal sense, this is very similar to Equation 9, which described loudness matches in the presence of a constant level of noise in the experiment by S. S. Stevens and Guirao (1967). The correspondence becomes even more striking when it is noted that in Figure 1 it was demonstrated that click-evoked voltage at the cochlea of the guinea pig is a linear function of the cube root of sound pressure and that in Figure 4 it was shown that scaled loudness in humans is also a linear function of the cube root of sound pressure. Since the effect of damage to the outer hair cells can be represented by the same form of equation as the effect of a masking noise in human psychophysical judgments, it is not unreasonable to suggest that a masking noise may temporarily disable the outer hair cell population in humans.

\section{THE GENERAL EQUATION}

To write a general equation, we first need to examine the question of the proper physical units to employ. The stimulus in audition is usually expressed in terms of pressure or occasionally in terms of energy, which is usually proportional to the square of pressure. The stimulus in vision is commonly expressed in terms of energy. The stimulus for touch is usually given in terms of amplitude displacement and for taste in terms of weight of solute per liter of solution or per $100 \mathrm{cc}$ of solvent.

In the equations for external and internal masking in vision, the use of energy units required an additional exponent. It would, therefore, produce a more simple equation if the stimulus in vision were expressed as pressure. The stimulus for taste in Equation 17 is expressed in units of weight of solute with respect to weight of solvent. This is in contrast to the usual chemical practice of specifying concentration in terms of number of gram molecular weights per volume of solution. There is no a priori reason for favoring either convention and, in dilute solutions, the two concentrations are proportional. However, for the definition of concentration employed in Equation 17, osmotic pressure is more nearly proportional to the concentration. Therefore, stimulus pressure again appears to be the optimum unit for predicting sensory magnitude.

The general equation for magnitude of response as a function of pressure under all conditions can be written as:

$$
S=R\left(P^{q}-P_{o}^{q}\right) 10^{-c P}
$$

where $\mathrm{S}$ is the magnitude of the response, $\mathrm{P}$ is the pressure of the stimulus, and $P_{0}$ is the threshold pressure of the stimulus. $R$ and $c$ are empirical constants. 
Most of the evidence seems to support a value of 0.29 for the exponent for vision when the stimulus is expressed in terms of energy; therefore, q would be 0.58 for vision if the stimulus is considered in terms of pressure. Physiological and some psychophysical experiments support a value of 0.33 for audition, and the results of scaling studies are also in agreement if the hypothesis of dual processes for loudness is accepted. There is some doubt concerning the exponent for taste, since it may vary for different sensory qualities and even for different testing procedures. There is general agreement that it is probably greater than unity, and the Stevens data depicted in Figure 8 suggest an exponent of 1.33. The data on vibrotaction illustrated in Figure 2 suggest an exponent of 0.50 , but this value should be considered tentative. The value of $R$ is a function of the choice of units for $S$, the level of adaptation, and the magnitude of an external masker.

In vision, the data of J. C. Stevens and S. S. Stevens (1963) show that the $R$ constant becomes smaller as the level of light adaptation increases. In touch, the data of Gescheider and Wright (1968) indicate that the $\mathbf{R}$ constant becomes smaller as the state of tactual adaptation increases. It would be anticipated that similar effects would appear in audition and in taste.

In audition, the results of Pollack (1949) demonstrate that the $\mathbf{R}$ constant is a decreasing exponential function of the noise-to-signal pressures. The data of S. S. Stevens and Guirao (1967) suggest that, under certain conditions, this constant may be a decreasing function of the noise pressure alone, but this effect is not consistent with the body of research on external masking in audition. On the contrary, the results of Hellman (1978), Hellman and Zwislocki (1964), and Scharf (1964) support the premise that $R$ is a function of the noise-to-signal pressure ratio.

In taste, the results of Beebe-Center, Rogers, Atkinson, and O'Connell (1959) suggest that the $\mathbf{R}$ constant is a decreasing function of a power of the osmotic pressure of the masker concentration. The relationship between $\mathbf{R}$ and masking in touch could not be established.

In vision, the relationship between $R$ and characteristics of the masker is complicated by the component of excitation contributed by the noise source. By an algebraic rearrangement of the right-hand side of Equation 12, we can express $R$ as:

$$
R=r\left[1+K_{3}\left(L_{n} / L_{s}\right)^{0.79}\right] 10^{-K_{1} \sqrt{L_{n} / L_{s}}} .
$$

The function of the $r$ constant is to adjust for the units of S. If the noise-to-signal ratio is maintained at a constant value, then the term within brackets is a linear function of the cotangent of the angle between the signal and noise sources. If this angle is kept constant, then the term within brackets is a lin- ear function of the 0.79 power of the ratio of the noise and signal luminances. If the noise-to-signal ratio is maintained at a constant value, then the magnitude of the exponential term will be an exponential function of the cotangent of the angle between the signal and noise sources. If this angle is kept constant, then the exponential term will be an exponential function of the square root of the ratio of the noise and signal luminances.

Although this relationship appears to be complex, it should be noted that when both the noise-to-signal ratio and the angle between noise and signal sources is constant, then $R$ will be a constant. Under this condition, $\mathrm{S}$ will be a simple linear function of a power of the physical stimulus and an equation similar to (3) will emerge. Except for values of $L_{s}$ close to the absolute threshold, the subjective brightness ratio of $L_{s}$ and $L_{n}$ will remain invariant with changes in $L_{s}$.

In vision, the $P_{0}$ constant increases as the level of light adaptation increases. In touch, the $P_{0}$ constant increases as the state of tactual adaptation increases. In both of these senses, there is a simple relationship in which $P_{0}$ is a linear function of the reciprocal of $\mathbf{R}$.

In vision, $P_{0}$ is an inverse function of the noise-tosignal ratio and the separation between the two sources under conditions of masking. In audition, $P_{0}$ is the absolute threshold in the absence of noise and is invariant with changes in the level of a masking source.

\section{REFERENCES}

Atxinson, W. H. Electrophysiological evidence for Stevens' power law at the medial geniculate of the cat. Brain Research, $1976,109,175-178$.

Atkinson, W. H. Dual processes for loudness (Paper presented at the 93rd meeting of the Acoustical Society of America). Journal of the Acoustical Society of America, 1977, 61, Suppl. 1, B11.

Atkinson, W. H. Click-evoked response at the superior colliculus of the cat. Experimental Neurology, 1978, 62, 501-509.

AtKInson, W. H., \& WARD, L. M. On the internal consistency of magnitude estimations. Perceptual and Motor Skills, 1972, 35, 903-913.

Bartoshuk, L. M., \& Cleveland, C. T. Mixtures of substances with similar taste. A test of a psychophysical model of taste mixture interaction. Sensory Processes, 1977, 1, 177-186.

Beebe-Center, J. G., Rogers, M. S., Atkinson, W. H., \& O'Connell, D. N. Sweetness and saltiness of compound solutions of sucrose and $\mathrm{NaCl}$ as a function of concentration of solutes. Journal of Experimental Psychology, 1959, 57, 231-235.

Borg, G., Diament, H., Strom, L., \& Zotererman, Y. The relation between neural and perceptual intensity: $A$ comparative study of the neural and psychophysical response to taste stimuli. Journal of Physiology, 1967, 192, 13-20.

Dallos, P., \& WANG, C.-Y. Bioelectric correlates of kanamycin intoxication. Audiology, 1974, 13, 277-289.

Diamond, A. L. Foveal simultaneous brightness contrast as a function of inducing- and test-field luminances. Journal of Experimental Psychology, 1953, 45, 304-314.

Diamond, A. L. Foveal simultaneous contrast as a function of inducing-field area. Journal of Experimental Psychology, 1955, s0, 144-152.

DiaMond, A. L. Simultaneous brightness contrast as a function of 
test-field area. Journal of Experimental Psychology, 1962, 64, 336-345.

Fechnen, G. T. Elemente der Psychophysik. Leipzig: Breitkopf \& Härterl, 1860.

Fry, G. A., \& AlPERn, M. The effect of a peripheral glare source upon the apparent brightness of an object. Journal of the Optical Society of America, 1953, 43, 189-195.

Fuortes, M. G. F. Electrical activity of cells in the eye of Limulus. American Journal of Ophthalmology, 1958, 46, 210-233.

Gescheiden, G. A. Psychophysics: Method and theory. Hillsdale, N.J: Erlbaum, 1976.

Gescheider, G. A., \& Wright, J. H. Effects of sensory adaptation on the form of the psychophysical magnitude function for cutaneous vibration. Journal of Experimental Psychology, $1968,77,308-313$.

Hawkins, J. E., \& Stevens, S. S. The masking of pure tones and of speech by white noise. Journal of the Acoustical Society of America, 1950, 22, 6-13.

HeinemanN, E. G. Simultaneous brightness induction as a function of inducing and test field luminances. Journal of Experimental Psychology, 1955, 50, 89-96.

Hellman, R. P. Dependence of loudness growth upon skirts of excitation patterns. Journal of the Acoustical Society of America, 1978, 63, 1114-1119.

Hellman, R. P., \& Zwislocki, J. Loudness function of a $1000 \mathrm{cps}$ tone in the presence of masking noise. Journal of the Acoustical Society of America, 1964, 36, 1618-1627.

Horeman, H. W. Relations between brightness and luminance under induction. Vision Research, 1965, 5, 331-340.

Lelbowitz, H., Mote, F. A., \& ThURLow, W. R. Simultaneous contrast as a function of separation between test and inducing fields. Journal of Experimental Psychology, 1953, 46, 453-456.

Lochner, J. P. A., \& Buraer, J. F. Form of loudness function in the presence of masking noise. Journal of the Acoustical Society of America, 1961, 33, 1705-1707.

Marks, L. E. Brightness as a function of retinal locus. Perception \& Psychophysics, 1966, 1, 335-341.

MArks, L. E. Brightness as a function of retinal locus in the light adapted eye. Vision Research, 1968, 8, 525-535.

Marks, L. E. Sensory processes: The new psychophysics. New York: Academic Press, 1974. (a)

MArks, L. E. On scales of sensation: Prolegomena to any future psychophysics that will be able to come forth as a science. Perception \& Psychophysics, 1974, 16, 358-376. (b)

Marks, L. E., \& Stevens, J. C. The form of the psychophysical function near threshold. Perception \& Psychophysics, 1968, 4, 315-318.

Moskowitz, H. R. Ratio scales of sugar sweetness. Perception \& Psychophysics, 1970, 7, 315-320.

Moskowitz, H. R. Intensity scales for pure tastes and for taste mixtures. Perception \& Psychophysics, 1971, 9, 51-57.

Pfapfmann, C. Gustatory nerve impulses in rat, cat, and rabbit. Journal of Neurophysiology, 1955, 18, 429-440.

Platenu, J. A. F. Sur la mesure des sensations physiques, et sur la loi lie l'intensité de ces sensations à l'intensité de la cause excitante. Bulletins de l'Academie Royale des Sciences, des
Lettres, et des Beaux-Arts de Belgique, 1872, 33, 376-385.

Pollack, I. The effect of white noise on the loudness of speech of assigned average level. Journal of the Acoustical Society of America, 1949, 21, 255-258.

ReEves, $P$. The response of the average pupil to various intensities of light. Journal of the Optical Society of America, 1920, 4, $35-43$.

Robinson, D. W., \& Dadson, R. S. A redetermination of the equal-loudness relations for pure tones. British Journal of Applied Physics, 1956, 7, 166-181.

Rodieck, R. W., \& Stone, J. Analysis of receptive fields of cat retinal ganglion cells. Journal of Neurophysiology, 1965, 28, 833-849.

Scharf, B. Partial masking. Acustica, 1964, 14, 16-23.

Steinberg, J. C., \& Gardner, M. B. The dependence of hearing impairment on sound intensity. Journal of the Acoustical Society of America, 1937, 9, 11-23.

Stevens, J. C. Brightness function: Binocular vs. monocular stimulation. Perception \& Psychophysics, 1967, 2, 451-454.

Stevens, J. C., \& Stevens, S. S. Brightness function. Effects of adaptation. Journal of the Optical Society of America, 1963, 53, 375-385.

Stevens, S. S. On the brightness of lights and the loudness of sounds. Science, 1953, 118, 576.

Stevens, S. S. Psychophysics of sensory function. In W. A. Rosenblith (Ed.), Sensory communication. Cambridge, Mass: M.I.T. Press, 1961. (a)

Stevens, S. S. To honor Fechner and repeal his law. Science, $1961,133,80-86$. (b)

Stevens, S. S. Power group transformations under glare, masking and recruitment. Journal of the Acoustical Society of America, 1966, 39, 725-735.

Stevens, S. S. Sensory scales of taste intensity. Perception \& Psychophysics, 1969, 6, 302-308.

Stevens, S. S. Neural events and the psychophysical law. Science, 1970, 170, 1043-1050.

Stevens, S. S. Psychophysics: Introduction to its perceptual, neural, and social prospects. New York: Wiley, 1975.

Stevens, S. S., \& Diamond, A. L. Effect of glare on the brightness function for a small target. Vision Research, 1965, 5, 649-659.

Stevens, S. S., \& Guirao, M. Loudness functions under inhibition. Perception \& Psychophysics, 1967, 2, 459-465.

Tens, D. C., Eldredge, D. H., \& Davis, H. Cochlear responses to acoustic transients: An interpretation of whole-nerve action potentials. Journal of the Acoustical Society of America, 1962, 34, 1438-1459.

Werblin, F. S. Adaptation in a vertebrate retina: Intracellular recording in Necturus. Journal of Neurophysiology, 1971, 34, 228-241.

Yilmaz, H. Perceptual invariance and the psychophysical law. Perception \& Psychophysics, 1967, 2, 533-538.

(Manuscript received July 14, 1980; revision accepted for publication September 30,1981 .) 